\title{
Mathematics in paper - from fiber suspension fluid dynamics to solid state paper mechanics
}

\author{
Jari Hämäläinen ${ }^{1 *}$, Taija Hämäläinen², Teemu Leppänen ${ }^{3}$, Heidi Niskanen ${ }^{4}$ and Joonas Sorvari
}

"Correspondence:

jari.hamalainen@lut.fi

${ }^{1}$ Centre of Computational

Engineering and Integrated Design

(CEID) and Department of

Mathematics and Physics,

Lappeenranta University of

Technology (LUT), P.O. Box 20,

Lappeenranta, 53851, Finland

Full list of author information is

available at the end of the article

\begin{abstract}
In papermaking process the base paper is formed by starting from low concentration suspension. Depending on process stage different models can be utilized for better understanding and performance of the process. A short general overview of fluid dynamics related to papermaking process is given as well as new approaches including fiber network modeling and continuum mechanical fracture simulations. The fluid dynamical results presented illustrated some paper machine wet end phenomena while the solid state modeling approaches can be exploited from drying section of a paper machine to the several converting processes.
\end{abstract}

Keywords: papermaking; multi-phase flow; fiber orientation; fiber flocculation; solid mechanics; fiber network; rheology of paper; Computational Fluid Dynamics (CFD); Finite Element Analyses (FEA)

\section{Introduction}

Paper is made from wood fibers with some chemicals and fillers. Papermaking starts from wood handling and stock preparation. Fibers are mixed in water in a low concentration (order of 1\%) when entering the first unit process of a paper machine, that is, the headbox (see Figure 1). Fibers tend to align with streamlines of a carrying phase water flow and they form fiber flocs in favorable flow conditions. By means of turbulence, both fiber orientation and flocculation can be controlled - stronger turbulence leads to more random orientation and less flocculated fiber suspension flow, which, in turn, influences the fibrous structure of the finished paper. The strength properties of the paper depend strongly on fiber orientation, which also determines the dimensional stability of the paper. Fiber flocs make denser 'spots' in a paper sheet, which may cause some variation in ink absorption, for example. Therefore, the control of fluid dynamics in the beginning of the papermaking process is a key issue in papermaking. Furthermore, the finished paper with its different end-use purposes can be studied by using mathematical modeling based on either fiberlevel network models or on the continuum approach. These aspects are discussed more detailed in this paper than in essay [1].

The paper is organized as follows: The second section deals with specific features of fiber suspension flows, namely fiber orientation and flocculation and how they can be modeled. The third section is devoted to the microscopic level solid mechanics of paper as a fiber network. Finally, the last section discusses the rheology of paper and its macroscopical modeling.

○2014 Hämäläinen et al.; licensee Springer. This is an Open Access article distributed under the terms of the Creative Commons Attribution License (http://creativecommons.org/licenses/by/2.0), which permits unrestricted use, distribution, and reproduction in any medium, provided the original work is properly cited. 
Figure 1 Paper machine headbox.
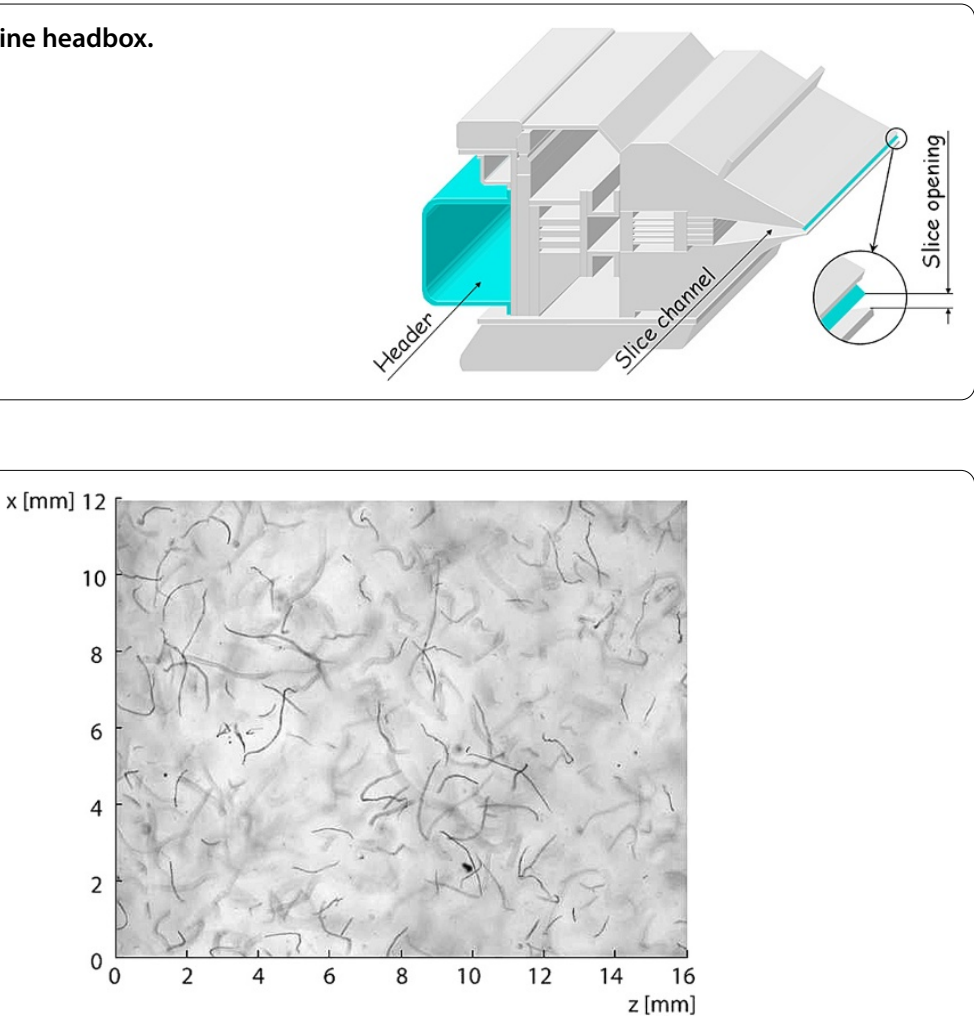

Figure 2 Fibers in a flow.

\section{Fluid dynamics of fiber suspension flows}

\subsection{Fiber orientation and flocculation}

Both fiber orientation and fiber flocculation are characteristic fluid dynamical phenomena of fiber suspension flows at low concentrations in the headbox and the forming section. The main purpose of a headbox is to create a thin and even jet which then enters the forming section. In the headbox, the suspension is mixed in the turbulence generator and then accelerated in the contracting slice channel. After the slice opening, the jet enters into free air and hits the moving fabrics called "wires", where the water removal and the formation of the fiber network are initiated. Subsequent water removal processes by pressing and drying make paper more solid, but the basic fibrous structure of paper does not change noticeably.

The structure of paper can be characterized by numerous mechanical properties. Fiber orientation describes how the fibers are aligned in the flow and finally in the finished paper as a fibrous network. Fibers are mainly aligned with streamlines of the headbox fluid flow; more precisely, the alignment of fibers forms a varying distribution which depends on the dominating flow conditions. A measured flow field with fibers is presented in Figure 2. Many mechanical properties of paper are affected by fiber orientation, for example, the strength properties of paper as it is stronger in the fiber direction than in the cross direction. Further, moisture changes cause fibers to swell, leading to cockling and curling of the finished paper. Therefore, headbox flows need to be controlled precisely so as not to cause harmful cross-directional flows in the headbox, such as those illustrated in Figure 3 due to a non-even flow rate distribution from the tapered header. The second mechanical 
Figure 3 Illustration of fluid flows in a headbox.

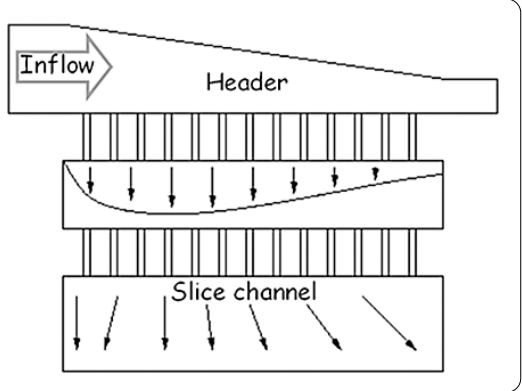

Figure 4 Basis weight variation called formation.

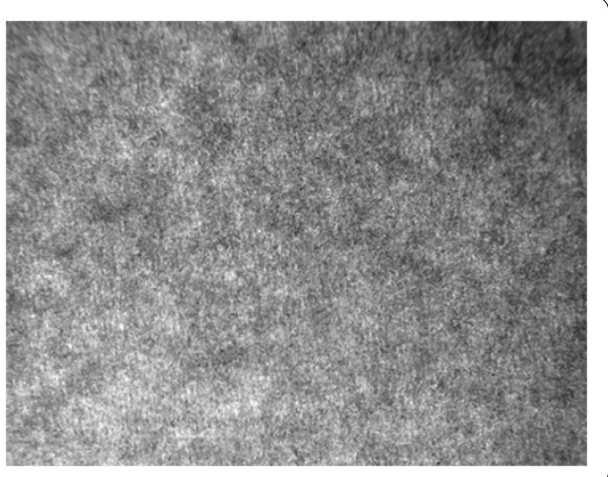

measure of paper is its basis weight defining how many grams it weights per square meter. Large-scale weight variations are controlled by headbox actuators, but small-scale variations are due to fiber flocculation (see Figure 4 for a photo of a paper sample in centimeter scale). Flocculation describes the tendency of fibers to form aggregates. Fibers used in papermaking tend to tangle together even in low concentration suspensions. This may affect the local variation of the solid mass distribution of paper and thereby causes undesired variance in the properties of the produced paper sheet. Non-uniformity of properties may induce problems in the later stages of papermaking or printing processes, as the unevenly distributed mass contains different quantities of water, and thus, expands unequally, or absorbs ink unevenly, deteriorating the quality of printed photos.

\subsection{Formulation of the fiber orientation probability distribution model}

When multiphase flows are concerned, there are namely two different methods to model the particles in such a flow. The first option is the Lagrangian approach, which models the motion of individual fibers. However, as accurate as it may be, this approach is computationally very expensive and thus it is not practical to be used in such a large scale flow as takes place in the headbox. The other option is the Eulerian approach, which takes into account an undefined number of fibers and predicts their statistical behavior. The latter one is the basic idea behind the fiber orientation probability distribution (FOPD) model.

The basis of the probability distribution modeling approach is the conservation of the probability flux and the use of diffusion-convection or the Fokker-Planck equation. This approach has been widely used (e.g. [2-5]) in studying the development of fiber orientation in contracting channel flows. As a result, the fiber orientation distribution may be obtained.

It is common to simplify the model and to consider only the planar orientation in vertical or horizontal planes. However, by doing so, one degree of freedom is lost and the results 
may not be as realistic as they would be when the motion of fiber is described in threedimensional space as it is in reality. Nevertheless, the model is able to illustrate the most probable orientation distribution in the headbox flow under turbulent conditions (e.g. [6, $7]$ ) and near walls [8]. The evolution of the fiber orientation distribution $\Psi$ can be written as

$$
\frac{\partial \Psi}{\partial t}-D_{t} \Delta \Psi-D_{r} \Delta_{S^{2}} \Psi+\nabla \cdot(\mathbf{v} \Psi)+\nabla_{S^{2}} \cdot(\mathbf{w} \Psi)=0
$$

where the notations $\Delta_{S^{2}}$ and $\nabla_{S^{2}}$ refer to the Laplace and divergence operators on a unit sphere, respectively. Furthermore, $\mathbf{v}$ is the velocity of the fluid and $\mathbf{w}$ is the rotational velocity of the fiber. Coefficients $D_{t}$ and $D_{r}$ are the translational and rotational diffusion coefficients respectively, describing the effects of velocity fluctuations on how the fibers are distracted from their state of orientation at a certain moment. It is usual to assume that there is no time-dependence, and thus, the first term of the equation can be ignored. The rotational velocity of the fiber is determined as (see e.g. [9-11])

$$
\mathbf{w}=\omega \mathbf{p}+\lambda \epsilon \mathbf{p}-\lambda\langle\mathbf{p}, \epsilon \mathbf{p}\rangle \mathbf{p}
$$

where $\lambda=\left(r^{2}-1\right) /\left(r^{2}+1\right)$ is a parameter related to the fiber aspect ratio $r=L / d, L$ and $d$ being the length and the diameter of the fiber, respectively, and $\mathbf{p}$ gives the orientation of the fiber at the surface of a unit sphere. The origin of the vector $\mathbf{p}$, defined as

$$
\mathbf{p}=\left(\begin{array}{c}
\cos \theta \sin \phi \\
\sin \theta \sin \phi \\
\cos \phi
\end{array}\right)
$$

is placed at the center of the fiber. The notation $\langle\cdot, \cdot\rangle$ refers to the inner product of the vectors. Furthermore,

$$
\boldsymbol{\epsilon}=\frac{1}{2}\left(\nabla \mathbf{u}+(\nabla \mathbf{u})^{T}\right) \quad \text { and } \quad \boldsymbol{\omega}=\frac{1}{2}\left(\nabla \mathbf{u}-(\nabla \mathbf{u})^{T}\right)
$$

are the strain rate and vorticity tensors, respectively. Resulting orientation distributions are illustrated in Figure 5. The figure shows the development of fiber orientation along a contracting channel achieved using a planar approximation of the model. Figure 6 shows the FOPD in three-dimensional surface, that is, a 3D solution for a $2 \mathrm{D}$ contracting channel and for one projected angle.

Figure 5 Fiber orientation distributions along a contracting channel. Modeled fiber orientation distributions. The channel outlet is located at $x=0.7 \mathrm{~m}$ and assumption of random orientation distribution is used at the channel inlet at $x=0 \mathrm{~m}$.

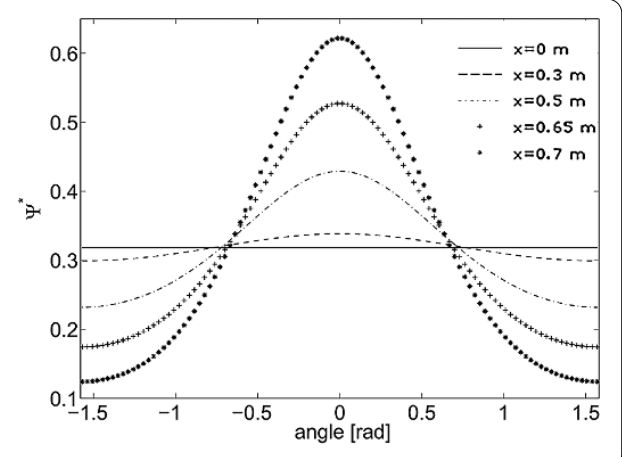


Figure 6 Visualization of the fiber orientation distribution.

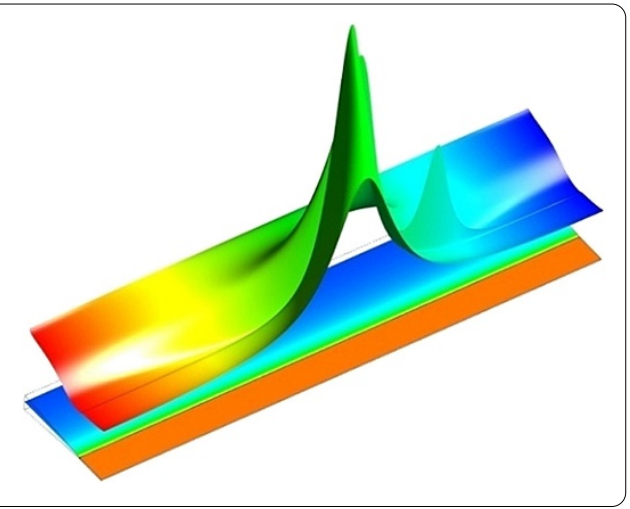

The earliest studies of non-spherical particles were conducted by Jeffery already in 1922 [12] when he derived the equations of motion for a single ellipsoid, non-Brownian particle in Newtonian fluid with simple shearing and showed that the particles execute rotational motion in periodic, closed orbits. Jeffery's work has been the basis for current modeling and has been widely applied. The model given above is based on the assumption that the fibers are rigid. However, it can also be used in describing the orientation of flexible fibers at least from a statistical point of view as discussed in [5] even though it does not take into account the flexibility of the fibers. Some studies have also taken into account the fiberfluid coupling through an orientation tensor including momentum exchange between the fiber and the fluid phases [13]. This type of approach to model the most probable orientation state of the fibers on different flow conditions has shown to be rather convenient when studying the flows of industrial scales. Naturally, there are some assumptions e.g. about the low enough concentration and rigidity of the fibers which does not necessarily meet the conditions in reality. The latter assumption is violated in the case of papermaking, where the wood fibers are long and flexible, thus causing additional complexity such as possible flocculation for the flow. However, the commonly taken approach gives practical information about the real-life phenomena present under the flows of papermaking and is of importance in trying to improve the papermaking process.

\subsection{Modeling of fiber floc dynamics by using a population balance approach}

Fiber flocculation has not been widely studied due to the challenges it creates both for measuring and computational methods. The simplest models treat fiber suspension as a one phase flow ([14-16]) although the interaction of the two fibers should be taken into account, as shown in [17] when comparing these results to measurements [18]. More accurate models $[19,20]$ use the Eulerian two-phase flow approach and are based on the population balance model also taking into account the occurrence of flocs of different sizes.

Modeling of fiber flocculation inside the headbox requires taking into account the twophase nature of the flow and the interactions between the carrying phase, water, and the dispersed phase, flocs. Moreover, to describe the real situation in the paper machine headbox and in the forming section, one has to model not only flocs of one size, but several sizes. This can be done by using the population balance approach

$$
\frac{\partial}{\partial t} n(v, t)+\frac{\partial}{\partial x^{i}}\left(U^{i}(v, t) n(v, t)\right)=B_{B}-D_{B}+B_{C}-D_{C},
$$


where $n(v, t)$ represents the number density of particles of size $v$ at time $t$ and $B_{B}, D_{B}$, $B_{C}$ and $D_{C}$ represent the birth rate due to the break-up of larger particles, the death rate due to the break-up into smaller particles, the birth rate due to the coalescence of smaller particles, and the death rate due to the coalescence with other particles, respectively. The carrying phase velocity field is denoted by $U . B_{B}, D_{B}, B_{C}$ and $D_{C}$ are defined as

$$
\begin{aligned}
& B_{B}=\int_{v}^{\infty} g(v ; s) n(s) d s, \\
& D_{B}=n(v) \int_{0}^{v} g(v ; s) d s, \\
& B_{C}=\frac{1}{2} \int_{0}^{v} Q(v-s ; s) n(v-s) n(v) d s, \\
& D_{C}=n(v) \int_{0}^{\infty} Q(v ; s) n(s) d s,
\end{aligned}
$$

where $g(v ; s)$ and $Q(v ; s)$ represent the specific break-up rate and the specific coalescence rate, respectively. For the break-up and coalescence models, the equations developed for bubble flows $[21,22]$ can be used, if the model parameters are adequately tuned and verified with measurements.

As the physical characteristics of wood fibers used in papermaking may vary significantly depending on the paper grade produced, it is intuitively clear that flocculation phenomena must depend on fiber characteristics. When thinking about a fiber network formed of long, flexible fibers or a network of short, stiff fibers, it is easy to see that breaking the network of long fibers requires more force than the short fiber case: If the concentrations of these two suspensions are the same, the suspension of long fibers has more interfiber connections to be broken. A practical approach to study this characteristic is an old concept - the crowding factor [23] defined as

$$
N_{\mathrm{cf}}=\frac{2}{3} C_{\nu}\left(\frac{L}{d}\right)^{2} \approx \frac{5 C_{m} L^{2}}{\omega}
$$

where $C_{v}$ is the volumetric concentration, $L$ is the average fiber length, $d$ is the average fiber diameter, $C_{m}$ is the pulp concentration, and $\omega$ is the fiber coarseness. The crowding factor represents the number of fibers within the rotational sphere of influence of a single fiber. It was shown in [19] that the internal strength of flocs is related to this factor.

The population balance approach allows the detailed study of the development of the floc size distribution, as shown in Figure 7. This example demonstrates the dynamics of a floc population of ten flow size groups between 1 and $10 \mathrm{~mm}$ in a headbox contracting channel. It shows that the smaller flocs are coalescing and their number density decreases whereas the number density of the largest flocs increases. For more detailed analyses, we refer to [19].

When fiber flocculation is modeled further in the paper machine in the forming section, more physical couplings are needed. In the forming section, most of the water included in the suspension is removed with different types of suction elements and the fiber concentration is significantly increased. This means more fiber-fiber interactions and less freedom for fibers to move. In addition, when the water is removed through the moving wires, the fiber network starts building up, further increases the resistance of the wires 

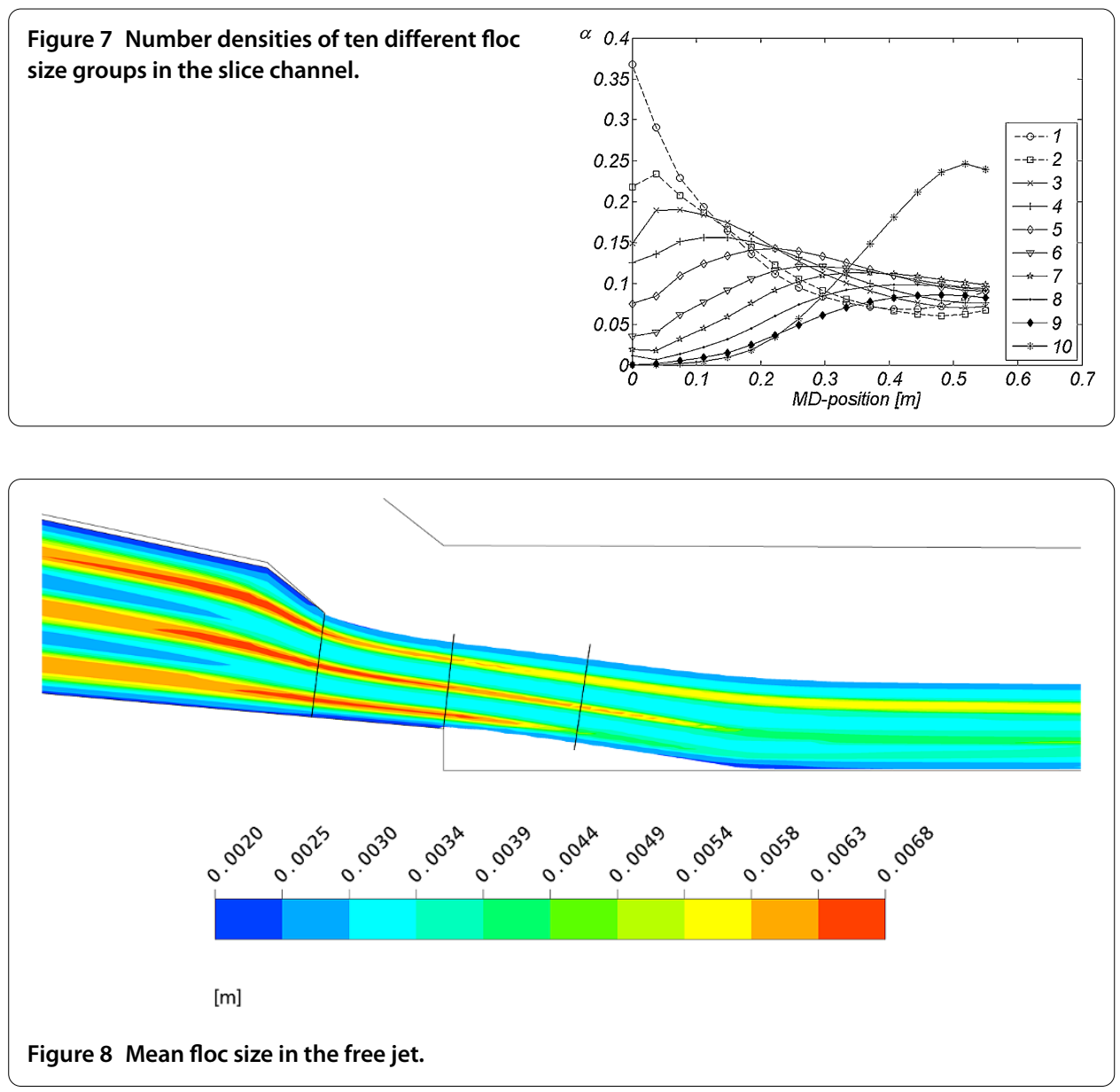

and thus hinders the water removal. This effect can be taken into account by adding the solids pressure force [24]

$$
F_{d, i}^{\mathrm{SP}}=-G_{0} e^{C_{\mathrm{SP}}\left(\alpha_{d}-\alpha_{d, \max }\right)} \frac{\partial \alpha_{d}}{\partial x_{i}}
$$

to the momentum equation of the dispersed phase. In Eq. (11) $G_{0}$ and $C_{\mathrm{SP}}$ are the reference elasticity modulus and the compaction modulus, respectively, and $\alpha_{d}$ and $\alpha_{d \text {,max }}$ represent the local volume fraction of the fibrous phase and the maximum packing parameter, respectively. Now by selecting suitable value for the coefficient $C_{\mathrm{SP}}$, the solids pressure gradient is only activated in the regions close to the maximum packing.

As discussed earlier in this paper, the flocculated state of the suspension is inherited to the variation of the solid mass distribution of the paper produced. Taking into account the phenomena in the forming section, the evolution of the fiber floc size can also be connected to the solid mechanical modeling of the finished paper sheet [20]. The shear forces and the water removal have significant effect on the floc size of the suspension, as illustrated in Figure 8. The figure illustrates the computational domain including the contracting channel with some turbulence lamellae causing the wakes in the flow, and the free jet from the headbox to the moving wire.

The following sections continue from the paper web obtained in the forming section and further dewatering in the papermaking process. 


\section{Paper as fibrous material}

\subsection{Paper physics}

Stress-strain curves yield important information on materials behavior. For a typical paper grade, such as newsprint, the stress-strain curve is characterized by an initial linear region which is followed by a non-linear region. The transition from the linear to the non-linear region takes place smoothly. In engineering literature, the transition point is known as the proportional limit. The proportional limit is commonly associated with the yield limit which is the point in the stress-strain curve after which plastic or permanent strains are developed. In the non-linear region gradient of the curve decreases but is still positive. The degree of non-linearity depends on humidity conditions. In dry conditions, the curve is rather linear even after the proportional limit. However, in humid conditions clear strain hardening is observed. In this sense, paper is brittle in dry conditions and ductile in humid conditions. The stress-strain curve depends also on the rate of loading. Both the elastic modulus and tensile strength increase with the strain rate. The viscous nature of paper can also be observed from a relaxation or creep test.

Since paper is a fibrous network in which fibers are connected by hydrogen bonds, we may ask what is the influence of fiber and bond properties on the mechanical properties of a paper sheet. It is intuitively clear that the elastic modulus of paper must depend on the elastic modulus of fibers. Due to the porous structure of paper and random orientation of fibers, the modulus is less than the corresponding modulus of fibers. In a seminal paper by Cox [25] a network theory for the tensile behavior of paper was presented. In the network model, long, straight and non-interacting fibers were considered. In Cox's theory, the elastic modulus of a paper sheet is given by $E=\frac{1}{3} \rho / \rho_{f} E_{f}$, where $E_{f}$ is the elastic modulus of fibers and $\rho$ and $\rho_{f}$ are densities of the sheet and the fibers, respectively. For highly bonded networks, Cox's theory seems to be in reasonable agreement with the experimental observations. However, for less dense networks the modulus can be lower than predicted by Cox's theory [26].

The non-linear irreversible response of a paper sheet under elongation has been attributed to the progressive disruption of inter-fiber bonds and to intra-fiber mechanisms. Rance [27] argued that the breaking of bonds results in increased deformation due to more localized stress concentrations. However, according to Alava [28], the inelasticity of paper arises from plastic deformations that take place in the fibers when microfibrils slide relative each other.

It is also still unclear whether the time-dependence of paper arises from fiber properties or from the behavior of inter-fiber bonds. In an in-depth review by Haslach [29], it was concluded that most likely the time-dependency is controlled by a mechanism which occurs within the inter-fiber bonds, namely the release of microcompression in the interfiber bonds.

Although there is currently no comprehensive theory of the inelasticity deformation of paper, there is consensus between paper physicists that the fracture strength of paper is governed by the failure of bonds. Only in highly bonded networks is the sheet strength determined by the tensile failure of fibers.

\subsection{Fiber network modeling}

It is obviously in the interest of papermakers to gain information on the relationship between the mechanical properties of paper and the constituent parts of paper. Extensive efforts have been made to construct realistic fiber networks and to simulate their behavior. 
Heyden [30] classifies network modeling approaches into uniform strain models, semianalytical models and to computer simulation models. In this paper, we are interested in the latter and especially in simulation models in which the finite element method (FEM) is utilized.

The first step in the fiber network modeling is to construct a realistic network, which is a challenging task especially if 3D structures are considered. The generation begins by placing fibers randomly inside a cell [30] or by depositing fibers on a rectangular surface one-by-one [31] until the desired network density or grammage is attained. Fiber parameters such as length, orientation and curvature may vary according to a probability distribution. In the network generation, geometric intersection or closest point problems are typically addressed in order to find possible inter-fiber bonding points. Free fibers or fiber clusters which are not connected to the rest of the network are typically removed from the network in order to obtain positive definite system matrix in finite element calculations. Network connectedness can be examined with aid of graph search algorithms [30]. The graph nodes are the intersection and boundary points and free fiber segments represent the graph edges. It should be noted that network systems are large. In an area of one square millimeter there are roughly 100-200 fibers, and every fiber has approximately 10-40 inter-fiber bonds. Furthermore, the number of the fibers increases quadratically with the area of the network. The size of the network is still the limiting factor when considering industrially important problems. We are still in the millimeter scale when dense three-dimensional networks are considered. To increase the size of the networks, efficient algorithms and coding methods are needed.

Network generation is only one part of the problem. In order to perform simulations, a finite element model of the generated network must be created. Although it is possible to model fibers with solid continuum elements [32], beam elements are typically used [30, 31] since they reduce the size of the discrete finite element system. Fibers are commonly assumed to follow the linearly elastic constitutive law [30], although inelastic models [32] can and should be used if extensive deformations are considered. Considering the physical behavior of paper networks, it seems that the proper modeling of inter-fiber bonding is critical in order to model paper networks realistically. For small deformations, ideal rigid bonding, as used in Cox's theory, may be adequate. However, to model the non-linear response or failure of a paper sheet, deformable bonding models which allow bond failure should be utilized. In Heyden's [30] network model, fibers are bonded together with an elastic spring, the stiffness and strength of which reduces in a stepwise manner until complete failure occurs. There has been growing interest to take into account the subsequent sliding of fibers after bond failure has occurred [31, 33]. The inter-fiber frictional sliding may be particularly important for wet networks since water breaks hydrogen bonds so that fibers can more easily slide against one another.

The resulting discrete finite element problem of fiber networks is typically very large and non-linear due to possible material and geometric non-linearity and frictional sliding. Thus, tools of high performance computing must be utilized.

\subsection{A fiber network model}

For illustrative purposes we consider here the problem of simulating the behavior of a fiber network in an extension test. Recently, Lavrykov et al. [32] have presented an alternative way to generate fiber networks. In their model, the sheet structure is obtained 
Figure 9 Example network geometry. Example of

a generated fiber network.

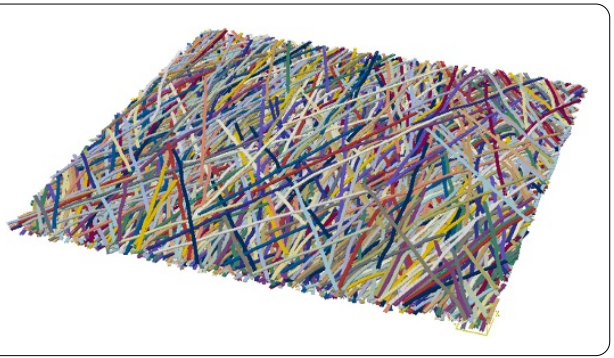

by compressing randomly placed fibers between two rigid plates. The compressing was simulated with the finite element method. A similar approach is used here.

In our deposition model, fibers are initially modeled as straight rectangular cuboids with a uniform length. The fibers are randomly placed on a plane and are not to allowed to intersect. At this stage, the fibers are parallel to the plane. The in-plane orientation of the fibers varies according to a uniform distribution. After fibers can no longer be placed on a given plane, we proceed to the next plane, and this procedure is continued until the desired grammage is attained. In other words, fibers are placed in a cell plane by plane. After the initial structure has been obtained we proceed to FEM simulation where we discretize the fibers with hexahedron elements and place the structure between two rigid plates. Then, the fibers are allowed to drop under gravity to the bottom stationary rigid plate. The upper rigid plate is moving and compresses the fibers so that the prescribed thickness of the network is attained. The forming of the sheet structure, i.e. dropping of the fibers and compression of the network, is simulated with aid of the FEM. In the simulation, fiber-tofiber contacts are taken into account. After we have obtained the initial sheet structure, fiber-to-fiber contact points are searched and in the contact points bonds are formed. Bonds are modeled as multiaxial springs that can fail if a given threshold value is reached. An example of a generated network is shown in Figure 9.

The network generation procedure was implemented in ABAQUS finite element software using Python scripting. In this way, we can combine finite element model making and simulations with useful numerical tools. In the initial intersection testing and in the search for fiber-to-fiber contact Gilbert-Johnson-Keerthi (GJK) collision detection algorithm [34] of convex objects is utilized. For a non-convex object, the same GJK algorithm can be used to detect the collision of the convex hulls of the objects. In the contact searching, bounding volume hierarchies and hierarchy traversal collision testing are used. In a bounding volume hierarchy, a geometric object is partitioned into a tree structure. The bounding volume hierarchy of a fiber is constructed using convex hulls. The root node is the convex hull of the fiber. At each node, the fiber part is subdivided into two nearly equal size parts and the convex hulls of the parts are stored in the child nodes. This procedure is continued until we end up in a structure in which the leaf nodes contain the convex hull of a single element. The fiber-to-fiber intersection tests are conducted using their bounding volume hierarchies. In the intersection tests, both hierarchies are descended simultaneously using a depth-first approach [35]. After all contacts have been found, the network connectedness is studied using the breadth-first search (BFS) strategy.

Figure 10 illustrates the effect of the bonding properties of fibers on the stress-strain behavior of a paper sheet. We simulated, using the FEM, an extension test using the same network but varied the bonding properties of fibers. The properties of the network are given in Table 1. If the bonds are rigid, the stress-strain curve is rather linear. The behavior 
Figure 10 Stress-strain curve of the network. Effect of bond properties on the stress-strain behavior of a paper sheet.

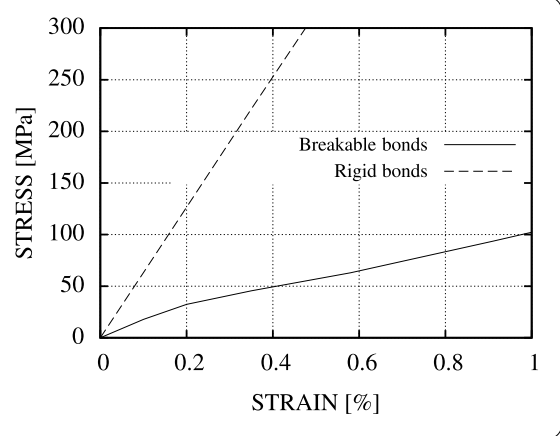

Table 1 Fiber network properties

\begin{tabular}{lll}
\hline Fiber length & {$[\mathrm{mm}]$} & 2 \\
Fiber width & {$[\mu \mathrm{m}]$} & 30 \\
Fiber thickness & {$[\mu \mathrm{m}]$} & 10 \\
Fiber basis weight & {$\left[\mathrm{g} / \mathrm{m}^{2}\right]$} & 8 \\
Elastic modulus of fibers & {$[\mathrm{GPa}]$} & 35 \\
Network size & {$\left[\mathrm{mm}^{2}\right]$} & $4 \times 4$ \\
Network thickness & {$[\mu \mathrm{m}]$} & 100 \\
Number of fibers & & 1,400 \\
\hline
\end{tabular}

(a)

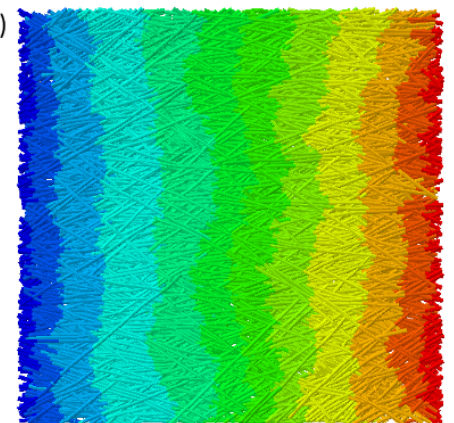

(b)

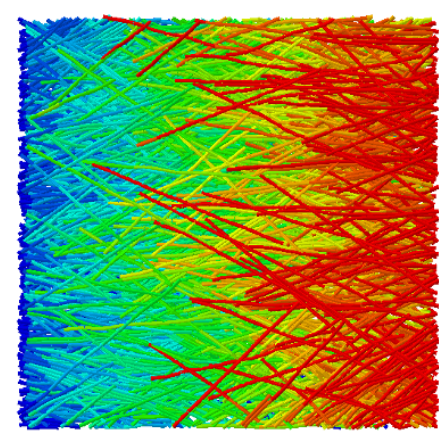

Figure 11 MD displacement fields. Effect of bond properties on the MD displacement fields. (a) Rigid bonds. (b) Stiff breakable bonds. Red color indicates maximum displacement, blue color minimum displacement.

is different from the case where the bonds are modeled with stiff breakable bonds in which case we see strain hardening as bonds deform and break. Figure 11 shows that for several fibers which are located near the loading edge, many of the bonds are broken so that the fibers are more free to move with the displaced edge.

\section{Paper as continuous material}

\subsection{Continuum description of paper}

At the macroscopic level, the paper structure can be considered as continuous solid material obeying the laws of continuum mechanics. The equilibrium equations together with constitutive laws form the basis of solid continuum mechanics. The continuum description of paper is a convenient approach to solve industrially relevant problems. However, paper is a heterogeneous and anisotropic viscoplastic material which makes it challenging to model even when the continuum description is used. In modeling applications, some simplifications are commonly needed. On a sufficiently large scale, paper can be consid- 


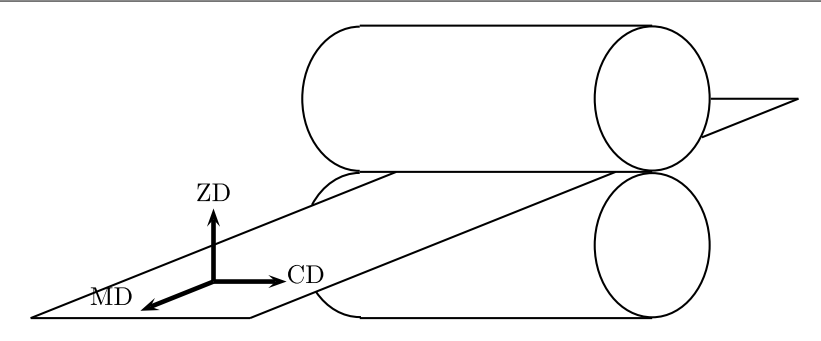

Figure 12 Principal material directions of paper. Principal material directions of machine made paper: MD, CD and ZD.

ered to exhibit homogeneous material behavior. In addition, the anisotropic behavior of paper is often handled by assuming paper to behave orthotropically. An orthotropic material has three orthogonal planes of symmetry which can be chosen as coordinate planes. In the case of machine made paper, the principal material directions coincide with MD, $\mathrm{CD}$ and the thickness direction (ZD), as illustrated in Figure 12. Paper is very orthotropic, being much stiffer in $\mathrm{MD}$ than in $\mathrm{CD}$ or $\mathrm{ZD}$. The value for the ratio between the elastic modulus in MD and CD is between 2 and 4 and for MD and ZD around 100.

Although the uniaxial inelasticity of paper has been long recognized [36] and is currently relatively well understood $[29,37]$, only very recently have well calibrated multiaxial inelastic constitutive models been proposed. Classical plasticity models have been proposed by Xia et al. [38], Mäkelä and Östlund [39] and more recently by Harryson and Ristinmaa [40]. Integral based viscoelastic constitutive models have been proposed by Uesaka et al. [41] and Lif [42]. Apparently, we are still missing a model which combines the viscous and plastic properties of paper.

The continuum description of paper is widely used in modeling applications. A typical application is FEM modeling related to papermaking or to printing processes. Examples include web runnability [43], calendering [44], winding [45] and printing nip mechanics [46].

\subsection{Modeling the fracture of paper}

As an example of an application of a continuum description, we consider modeling the fracture of paper. As the continuum description is used, paper is treated as a solid continuum. However, material properties are allowed to vary locally. To be precise, paper is divided into small volumes each having their own principal material directions. In addition, every volume has its own directional stress-strain behavior depending on, for example, the fiber orientation anisotropy, dry solids content and basis weight. More information about this method, the locally varying fiber orientation and fiber orientation anisotropy can be found from reference [47]. As an example, Figure 13 shows how the tensile strength depends on fiber orientation anisotropy $\xi$ and dry solids content $\beta$ in the example presented in this paper. A detailed and sophisticated presentation of material parameter dependencies is available in reference [48].

The failure model applied is a simplification and generalization of Hashin's theory [49, 50]. Hashin's theory was created for fiber-reinforced materials. It requires that the behavior of undamaged material is linearly elastic. It takes into account four different failure modes: fiber tension, fiber compression, matrix tension, and matrix compression. When 
Figure 13 Tensile strength of paper. Tensile strengths as a function of the fiber orientation anisotropy $\xi$ and dry solids content $\beta$ for the main direction (MD).

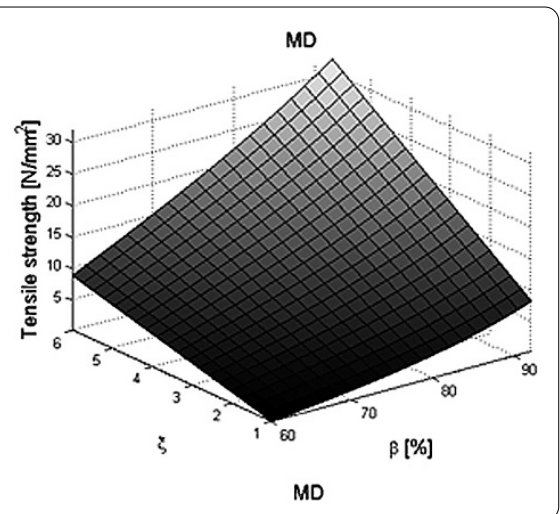

this theory is used, two different stages of the fracture process should be defined: damage initiation and damage evolution.

In the case of paper, the linear elasticity requirement in Hashin's theory is a substantial weakness; the stress-strain behavior of paper is commonly nonlinear and inelastic, as stated earlier. From that point of view, the failure model is a generalization of Hashin's theory since no restrictions are imposed for material behavior. Actually, if fracture simulations are considered, the essential issue is the determination of the tensile strength (and stress-strain behavior) dependencies (see Figure 13). Whereas in Hashin's theory four different failure modes are considered, in the failure model applied only two failure modes are considered; the failure of fibers and matrix are not separated. From that point of view, the present model is a simplification compared to Hashin's theory. Also the damage evolution is much simpler in the present model than in Hashin's theory: when the stress level reaches the tensile strength, the stiffness of the material is decreased to a thousandth. The failure model was implemented in the ABAQUS user subroutine USDFLD. Numerical problems were addressed by a two step stabilization process. The first step is directly controlled by the user and the second step is adaptively process controlled by ABAQUS.

Figure 14 presents a simulated fracture of a paper sample in an extension test. The sample was loaded in MD. The basis weight of the sample was $45 \mathrm{~g} / \mathrm{m}^{2}$ and the constant dry solids content and basis weight was assumed. The only variation taken into account was the variation of fiber orientation in all three directions: $\mathrm{MD}, \mathrm{CD}$ and $\mathrm{ZD}$. As can be seen from the MD strains in Figure 14, the location of the initial fracture is not a simple matter. It is not determined only by one location - also the surrounding areas of one location influences the stress level of the location. In addition, the whole stress-strain behavior varies in all directions. That is, a higher MD strain level in some location does not mean that the fracture starts from that location.

\section{Conclusions}

This paper aimed at giving a general view on mathematical modeling in papermaking. Many modeling challenges are excluded from this paper: Fluid-Structure Interactions (FSI) for the running paper web, coating of the paper web, the rheology of fluids and solids, heat transfer during the drying process, to mention a few. However, modeling approaches presented in this paper may be used separately or together in several paper production, converting or end-use situations. For example, by fluid dynamics the variation of fiber orientation and basis weight may be predicted and by solid mechanics the importance of these factors on paper behavior can be studied. The future work concentrates to the col- 

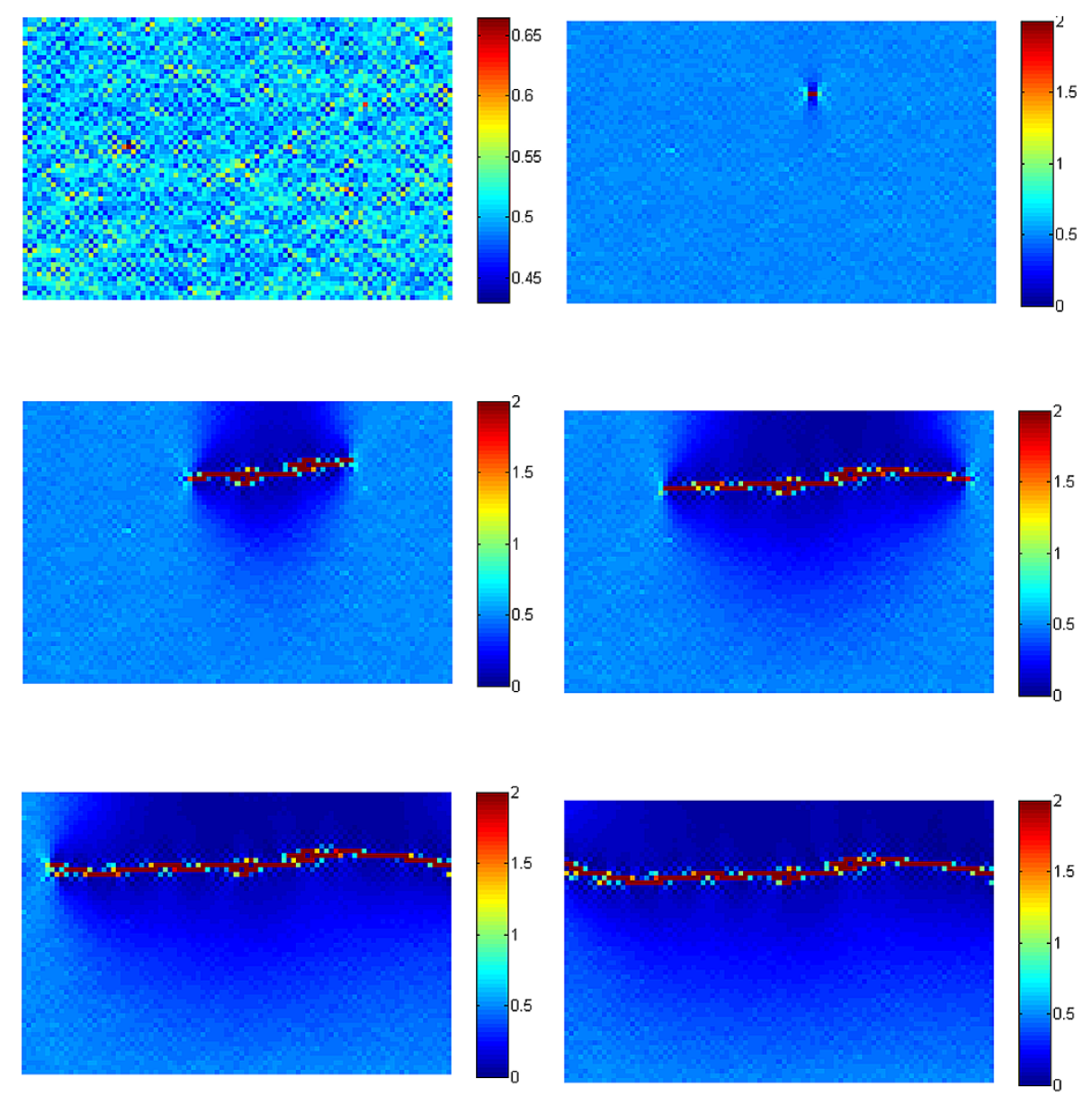

Figure 14 Simulated fracture process. Simulated MD strain during the fracture process when the variation of fiber orientation is taken into account. In figures, MD corresponds to the vertical direction and the fracture proceeds from left to right and from top to bottom. A $90 \%$ dry solids content was used in the simulation.

laboration of the presented modeling approaches, for example, stress-strain behavior used in continuum mechanical approach could be obtained by fiber network modeling.

\section{Competing interests}

The authors declare that they have no competing interests.

\section{Authors' contributions}

All authors contributed equally to the writing of this paper. All authors read and approved the final manuscript.

\section{Author details}

${ }^{1}$ Centre of Computational Engineering and Integrated Design (CEID) and Department of Mathematics and Physics, Lappeenranta University of Technology (LUT), P.O. Box 20, Lappeenranta, 53851, Finland. ${ }^{2}$ Metso Automation Oy, Vanha Porvoontie 229, Vantaa, 01380, Finland. ${ }^{3}$ LUT Savo Sustainable Technologies (Varkaus), Lappeenranta University of Technology, Osmajoentie 75, Varkaus, 78210, Finland. ${ }^{4}$ ELOMATIC, Itäinen Rantakatu 72, Turku, 20810, Finland.

Received: 28 February 2014 Accepted: 25 October 2014 Published: 11 Nov 2014

\section{References}

1. Hämäläinen J: Mathematics in paper. J Math Ind 2012, 2:2.

2. Olson JA: The motion of fibres in turbulent flow, stochastic simulation of isotropic homogeneous turbulence. Int J Multiph Flow 2001, 27:2083-2103.

3. Hyensjö M, Dahlkild A: Study of the rotational diffusivity coefficient of fibres in planar contracting flows with varying turbulence levels. Int J Multiph Flow 2008, 34:894-903. 
4. Krochak PJ, Olson JA, Martinez DM: The orientation of semidilute rigid fiber suspensions in a linearly contracting channel. Phys Fluids 2008, 20:073303.

5. Niskanen $\mathrm{H}$, Eloranta $\mathrm{H}$, Tuomela J, Hämäläinen J: On the orientation probability distribution of flexible fibres in a contracting channel flow. Int J Multiph Flow 2011, 37:336-345.

6. Olson JA, Kerekes RJ: The motion of fibres in turbulent flow. J Fluid Mech 1998, 377:47-64.

7. Olson JA, Frigaard I, Chan C, Hämäläinen J: Modelling turbulent fibre suspension flowing in a planar contraction: the one-dimensional headbox. Int J Multiph Flow 2004, 30:51-66.

8. Krochak PJ, Olson JA, Martinez DM: Near-wall estimates of the concentration and orientation distribution of a semi-dilute rigid fibre suspension in Poiseuille flow. J Fluid Mech 2010, 653:431-462.

9. Shin M, Koch DL: Rotational and translational dispersion of fibres in isotropic turbulent flows. J Fluid Mech 2005, 540:143-173.

10. Parsheh M, Brown M, Aidun C: On the orientation of stiff fibres suspended in turbulent flow in planar contraction. J Fluid Mech 2005, 545:245-269.

11. Phelps JH, Tucker CL III: An anisotropic rotary diffusion model for fiber orientation in short- and long-fiber thermoplastics. J Non-Newton Fluid Mech 2009, 156:165-176.

12. Jeffery GB: The motion of ellipsoidal particles immersed in a viscous fluid. Proc R Soc Lond A 1922, 102:161-179.

13. Krochak PJ, Olson JA, Martinez M: Fiber suspension flow in a tapered channel: the effect of flow/fiber coupling. Int J Multiph Flow 2009, 35:676-688.

14. Steen M: Turbulence and flocculation in fibre suspensions. PhD thesis. University of Trondheim, Norway; 1990.

15. Kuhn DCS, Sullivan PE: Analysis and measurement of the flocculation intensity of flowing pulp suspensions. In TAPPI Papermakers Conference; 2001

16. Plikas A, Kuhn DCS, Sullivan PE: A numerical model of fibre suspensions in a grid generated turbulent flow. In Proceedings of ASME FEDSM'00; 2000:991-996.

17. Hyensjö M: On fibre suspension flow modelling: mechanical fibre flocculation and fibre orientation. PhLic thesis. Royal Institute of Technology, Stockholm, Sweden; 2005.

18. Karema $\mathrm{H}$, Salmela J, Tukiainen $\mathrm{M}$, Lepomäki $\mathrm{H}$ : Prediction of paper formation by fluidisation and reflocculation experiments. In 12th Fundamental Research Symposium; 2001:559-589.

19. Hämäläinen T, Hämäläinen J: Fibre floc evolution model, part I: flocculation in a headbox. Nord Pulp Pap Res J 2010, 25(1):39-47.

20. Hämäläinen T, Hämäläinen J: Fibre floc evolution model, part II: influence of the jet-to-wire ratio to resulting fibre floc evolution in the initial drainage zone. Nord Pulp Pap Res J 2010, 25(1):48-55.

21. Luo H, Svendsen HF: Theoretical model for drop and bubble breakup in turbulent dispersions. A/ChE J 1996, 42(5):1225-1233.

22. Prince MJ, Blanch HW: Bubble coalescence and break-up in airsparged bubble columns. AlChE J 1990, 36(10):1485-1499.

23. Kerekes RJ, Schell CJ: Characterization of fibre flocculation regimes by a crowding factor. J Pulp Pap Sci 1992, 18(1):32-38.

24. Gidaspow D: Multiphase Flow and Fluidization. London: Academic Press; 1994

25. Cox HL: The elasticity and strength of paper and other fibrous materials. Br J Appl Phys 1952, 3:72-79.

26. Sampson WW: Materials properties of paper as influenced by its fibrous architecture. Int Mater Rev 2009, 54:134-156.

27. Rance HF: The formulation of methods and objectives appropriate to the rheological study of paper. Tappi J 1956, 2:104-115.

28. Alava M, Niskanen K: The physics of paper. Rep Prog Phys 2006, 69:669-723.

29. Haslach HW Jr.: The moisture and rate-dependent mechanical properties of paper: a review. Mech Time-Depend Mater 2000, 4:169-210

30. Heyden S: Network modelling for the evaluation of mechanical properties of cellulose fluff. PhD thesis. Lund University, Department of Mechanics and Materials; 2000.

31. Kulachenko A, Uesaka T: Direct simulations of fiber network deformation and failure. Mech Mater 2012, 51:1-14

32. Lavrykov S, Lindström SB, Singh KM, Ramarao BV: 3D network simulations of paper structure. Nord Pulp Pap Res $J$ 2012, 27:256-263.

33. Wilbrink DV, Beex LAA, Peerlings RHJ: A discrete network model for bond failure and frictional sliding in fibrous materials. Int J Solids Struct 2013, 50:1354-1363.

34. Gilbert EG, Johnson DW, Keerthi SS: A fast procedure for computing the distance between complex objects in three-dimensional space. IEEE J Robot Autom 1988, 4:193-203.

35. Mukundan R: Advanced Methods in Computer Graphics: With Examples in OpenGL. London: Springer; 2012.

36. Steenberg B: Paper as a visco-elastic body. Sven Papperstidn 1947, 50:127-140.

37. Coffin DW: The creep response of paper. In 13th Fundamental Research Symposium; 2005:651-747.

38. Xia QS, Boyce MC, Parks DM: A constitutive model for the anisotropic elastic-plastic deformation of paper and paperboard. Int J Solids Struct 2002, 39:4053-4071.

39. Mäkelä P, Östlund S: Orthotropic elastic-plastic material model for paper materials. Int J Solids Struct 2003 , 40:5599-5620.

40. Harryson A, Ristinmaa M: Large strain elasto-plastic model of paper and corrugated board. Int J Solids Struct 2008, 45:3334-3352

41. Uesaka T, Kodaja I, Okushima S, Fukuchi R: History-dependent dimensional stability of paper. Rheol Acta 1989, 28:238-245.

42. Lif J: In-plane hygro-viscoelasticity of paper at small deformations. Nord Pulp Pap Res J 2005, 20:139-149.

43. Lif J: Hygro-viscoelastic stress analysis in paper web offset printing. Finite Elem Anal Des 2006, 42:341-366.

44. Wikström M: Finite element modelling of calendering - some aspects of the effects of temperature gradients and structure inhomogeneities. J Mater Sci 1996, 31:3159-3166.

45. Ärölä K, von Hertzen R: An elastoplastic continuum model for a wound roll with interlayer slippage. Finite Elem Anal Des 2006, 42:503-517. 
46. Kariniemi M, Parola M, Kulachenko A, Sorvari J, von Hertzen L: Effect of blanket properties on web tension in offset printing. In Advances in Printing and Media Technology; 2010

47. Lipponen P, Erkkilä A-L, Leppänen T, Hämäläinen J: On the importance of in-plane shrinkage and through-thickness moisture gradient during drying on cockling and curling phenomena. In 14th Pulp and Paper Fundamental Research Symposium; 2009:389-436.

48. Erkkilä A-L, Leppänen T, Hämäläinen J: Empirical plasticity models applied for paper sheets having different anisotropy and dry solids content levels. Int J Solids Struct 2013, 50:2151-2179.

49. Hashin Z, Rotem A: A fatigue criterion for fiber-reinforced materials. J Compos Mater 1973, 7:448-464.

50. Hashin Z: Failure criteria for unidirectional fiber composites. J App/ Mech 1980, 47:329-334.

10.1186/2190-5983-4-14

Cite this article as: Hämäläinen et al.: Mathematics in paper - from fiber suspension fluid dynamics to solid state paper mechanics. Journal of Mathematics in Industry 2014, 4:14

\section{Submit your manuscript to a SpringerOpen ${ }^{\circ}$ journal and benefit from:}

- Convenient online submission

- Rigorous peer review

- Immediate publication on acceptance

- Open access: articles freely available online

- High visibility within the field

- Retaining the copyright to your article 